The 65th special feature "Fluorine Chemistry and Materials for Electrochemistry"

\title{
Electrochemical Properties of Carbon Paste Electrodes Modified with Fluorinated Materials
}

Takuya OKADA, Fumiki TAKAHASHI, Jiye JIN, and Hirosuke TATSUMI*

Department of Chemistry, Faculty of Science, Shinshu University, 3-1-1 Asahi, Matsumoto, Nagano 390-8621, Japan

* Corresponding author: tatsumi@shinshu-u.ac.jp

\section{ABSTRACT}

A carbon paste electrode (CPE) modified with polytetrafluoroethylene (PTFE) powder and perfluoropolyether (PFPE) oil was studied. These two fluorinated materials were simply mixed with graphite powder. The voltammetric behavior was compared with those of non-fluorinated materials, i.e., polyethylene (PE) powder and liquid paraffin (LP) oil. The CPE with PTFE and PFPE gave a wider polarizable potential window and a lower charging current than the non-fluorinated CPEs. When the ionic strength of the test solution was sufficiently low, the electrostatic interaction between the reactant and the fluorinated electrode surface was observed. The CPE with PTFE and PFPE was stable in a tetrahydrofuran (THF) solution, whereas other CPEs were not, due to the dissolution of the non-fluorinated materials.

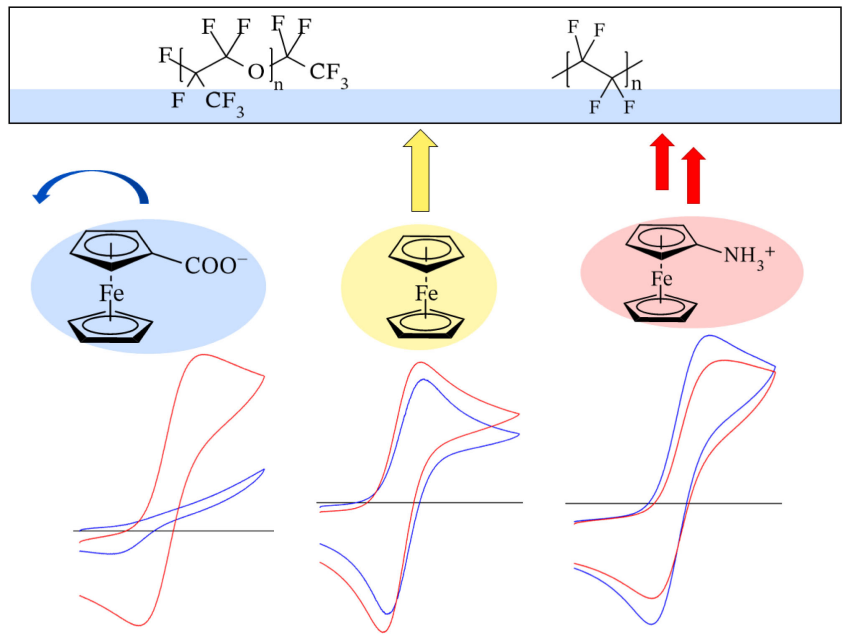

(C) The Author(s) 2020. Published by ECSJ. This is an open access article distributed under the terms of the Creative Commons Attribution Non-Commercial Share Alike 4.0 License (CC BY-NC-SA, http://creativecommons.org/licenses/by-nc-sa/4.0/), which permits non-commercial reuse, distribution, and reproduction in any medium by share-alike, provided the original work is properly cited. For permission for commercial reuse, please email to the corresponding author. [DOI: 10.5796/electrochemistry.20-65150].

Keywords : Carbon Paste Electrodes, Fluorinated Carbon Electrodes, Fluorous Solvents, Fluorocarbon Oils

\section{Introduction}

The surface modification of carbon electrodes by fluorine atoms has been studied in wide field of electrochemistry, expecting a new function of electrodes. ${ }^{1,2}$ For the purpose of electroanalysis, the fluorination of carbon electrodes has been achieved by means of bottom-up synthesis of fluorinated glassy carbon ${ }^{3,4}$ or nano-carbon film ${ }^{5}$ and $\mathrm{CF}_{4}$ plasma treatment ${ }^{6,7}$ or electrochemical pretreatment in perfluoroalkyl acid solution ${ }^{8}$ on boron-doped diamond. Unique electrochemical properties of fluorinated carbon electrode, such as an extraordinarily wide polarizable potential window due to the increase in the over potentials for hydrogen and oxygen evolution ${ }^{9}$ and the electrostatic interaction between the reactant and the electrode surface, ${ }^{5,7}$ have been reported. However, those fluorination methods often require rather complicated procedures with large and expensive instruments or environmentally unfriendly chemicals. Thus, a simple and inexpensive fluorination method is desirable.

Carbon paste electrodes (CPEs) have been widely used in electroanalysis since the invention by Adams. ${ }^{10}$ It has advantages of low background currents ${ }^{11}$ and simple modification by mixing functional materials, e.g. enzymes ${ }^{12-14}$ and nanoparticles. ${ }^{11}$ Nevertheless, only several reports have appeared on the modification of CPEs with fluorinated materials so far. Lindquist ${ }^{15}$ and Danielson and co-workers ${ }^{16-18}$ have used polychlorotrifluoroethylene (Kel-F) oil as the binder liquid. Some other composite electrodes have prepared by pressing graphite powder and $\mathrm{Kel}-\mathrm{F}^{19}$ or polytetra- fluoroethylene (PTFE) powder. ${ }^{20}$ However, these studies of the fluorinated CPEs or composite electrodes have focused only on the measurements in nonaqueous solutions. The details in the voltammetric behavior have not been studied comprehensively.

In this study, CPEs were prepared by simply mixing graphite powder, PTFE powder, and perfluoropolyether (PFPE) oil. To investigate the role of these fluorinated materials, the voltammetric results were compared with non-fluorinated materials, such as polyethylene (PE) powder and liquid paraffin (LP) oil. Unique properties were found at the fluorinated CPEs in aqueous and nonaqueous solutions.

\section{Experimental}

\subsection{Reagents}

PTFE powder (Dyneon TF9202Z) was kindly donated by 3M Japan. The average diameter of the powder was $4 \mu \mathrm{m}$. PFPE oil (Krytox GPL105, $1.93 \mathrm{~g} \mathrm{~cm}^{-3}$ density) was purchased from Maruwa Busssan K.K. (Tokyo, Japan). PE powder (Flo-beads LE1080, average diameter of $6 \mu \mathrm{m}$ ) was kindly donated by Sumitomo Seika Chemicals. LP oil with a density of $0.87 \mathrm{~g} \mathrm{~cm}^{-3}$ was purchased from Nacalai Tesque.

Aminoferrocene (TCI) and ferrocene (Sigma) were used without further purification and dissolved in ethanol. Ferrrocenecarboxylic acid (Wako) was mixed with equimolar amount of $\mathrm{KOH}$ and used as potassium salt of ferrocenecarboxylate. Other chemicals were 
obtained from Nacalai Tesque and used without further purification, except for tetrahydrofuran (THF), which was dried with molecular sieves $3 \mathrm{~A}$ before use.

\subsection{Carbon paste preparation}

Equal weights of graphite powder (Z-5F, Ito Graphite Co., Ltd, average diameter of $5 \mu \mathrm{m}$ ), the PTFE or PE powder, and the PFPE or LP oil were mixed well in an agate mortar. Typically, the composition was $0.1 \mathrm{~g}$ graphite, $0.1 \mathrm{~g}$ PTFE or PE, and $0.13 \mathrm{~mL}$ oil (weighs of PFPE and LP were $0.25 \mathrm{~g}$ and $0.11 \mathrm{~g}$, respectively). After mixing, the paste was packed in a carbon paste electrode holder (BAS 002210, $3 \mathrm{~mm}$ diameter) and its surface was polished on a weighing paper.

\subsection{Electrochemical measurements}

Cyclic voltammetry was carried out in a $0.1 \mathrm{M}$ or $1 \mathrm{mM}$ acetate buffer ( $\mathrm{pH} 5.1$ ) or $0.1 \mathrm{M}$ phosphate buffer solution ( $\mathrm{pH}$ 6.8). A platinum coil and an $\mathrm{Ag} / \mathrm{AgCl}(0.1 \mathrm{M} \mathrm{KCl})$ electrodes were used as the counter and the reference electrodes, respectively. For the measurements in nonaqueous solutions, $0.1 \mathrm{M}$ tetrabutylammonium perchlorate $\left(\mathrm{TBAClO}_{4}\right)$ was used as the supporting electrolyte, and the reference electrode was replaced by $\mathrm{Ag}\left(0.1 \mathrm{M} \mathrm{AgClO}_{4}+0.1 \mathrm{M}\right.$ $\mathrm{TBAClO}_{4}$ ) electrode.

Voltamograms were recorded by a potentiostat HECS 972S (Husou Seisakusyo, Kawasaki, Japan). The potentiostat was connected to a PC via an AD/DA converter and controlled by a laboratory-made $\mathrm{C}++$ program. All the experiments were performed at ambient temperature.

\section{Results and Discussion}

\subsection{Potential windows and charging currents}

The polarizable potential windows of the fluorinated CPEs were examined. Figure 1 compares the cyclic voltammograms of the CPEs with (a) PTFE and PFPE, (b) PTFE and LP, (c) PE and PFPE, and (d) PE and LP, in a pH 6.8 phosphate buffer solution after removing dissolved oxygen. When PTFE powder and PFPE oil were mixed in the carbon paste (curve a), the potential window was the widest of the four CPEs. Especially in the negative scan, the polarizable potential range reached $-2.0 \mathrm{~V}$ vs. $\mathrm{Ag} / \mathrm{AgCl}$. This is because of the slower electrode kinetics of the reduction of $\mathrm{H}^{+}$ion $\left(\mathrm{H}_{2}\right.$ evolution $)$ at the fluorinated electrode surface. Similar results of the extension of the polarizable potential window at fluorinated carbon electrodes by sputtering technique have been reported. ${ }^{7,9}$ When either of the powder or the oil was fluorinated (curves $b$ and c), the potential window was comparable to non-fluorinated CPE (curve d), probably due to the insufficient fluorination.

Small cathodic currents of $\mathrm{O}_{2}$ were observed at about $-1.2 \mathrm{~V}$ in curve a and $-0.9 \mathrm{~V}$ in curves $\mathrm{b}$, c, and d, although Ar gas was purged into the test solution for $60 \mathrm{~min}$ prior to each measurement. This may be derived from the $\mathrm{O}_{2}$ dissolved in the PFPE or LP oil. Carbon paste electrodes have been considered unsuitable for measurements at the negative potential range, because of the difficulty in removing $\mathrm{O}_{2}$ from the binder liquid. ${ }^{21}$ However, the cathodic signal of $\mathrm{O}_{2}$ at the CPE with PTFE and PFPE (curve a) was relatively insignificant, due to the slower electrode kinetics of $\mathrm{O}_{2}$ reduction.

It is known that the charging current of CPE is relatively small when compared to other electrodes, ${ }^{11,20}$ because the surface of CPE is partially covered with an insulator, which makes the exposed area of carbon reduced. Figure 2 compares the charging currents of the fluorinated CPEs in the potential range from 0 to $0.5 \mathrm{~V}$. The charging currents of the CPEs with PTFE powder (curves a and b) were smaller than PE powder (curves c and d). Especially, PTFE and PFPE (curve a) gave the smallest charging current. The parameters which can affect the capacitance at an electrode | solution interface

$$
\lceil 10 \mu \mathrm{A}
$$

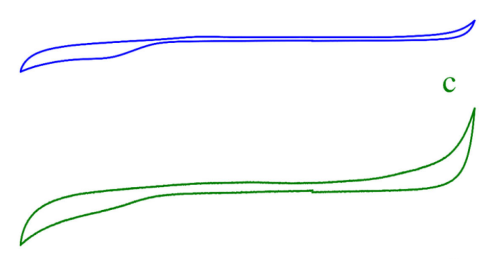

d

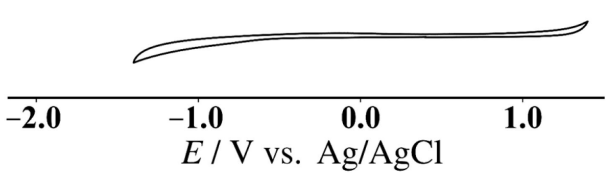

Figure 1. Cyclic voltammograms in $0.1 \mathrm{M}$ phosphate buffer (pH 6.8) at (a) PTFE-PFPE, (b) PTFE-LP, (c) PE-PFPE and (d) PE-LP CPEs. The buffer solution was deoxygenated with Ar gas for $60 \mathrm{~min}$. Scan rate: $0.2 \mathrm{~V} \mathrm{~s}^{-1}$.

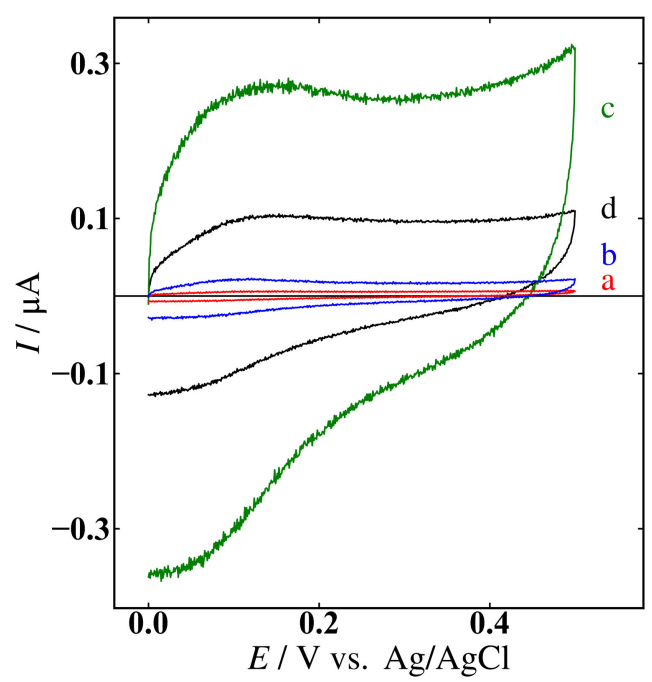

Figure 2. Cyclic voltammograms in $0.1 \mathrm{M}$ acetate buffer ( $\mathrm{pH} 5.1)$ at (a) PTFE-PFPE, (b) PTFE-LP, (c) PE-PFPE, and (d) PE-LP CPEs. Scan rate: $0.2 \mathrm{~V} \mathrm{~s}^{-1}$.

are the permittivity and the area. Since the relative permittivities of PTFE and PE (2.1 and 2.3, respectively) ${ }^{22}$ do not differ greatly, the difference in the charging current could be attributed to the exposed area of carbon. The particle sizes of the powder would be important, but we did not examine it here. Regarding the choice of the oil, it was interesting that PE and PFPE (curve c) gave the largest charging current of the four CPEs, whereas the smallest charging current was obtained with PTFE and PFPE (curve a). In the preparation, it was found that the PTFE-PFPE carbon paste had a smooth texture, while the PE-PFPE carbon paste had a rough texture. This may be due to the difference of wettability of these materials. The results can be interpreted as the difference of the roughness of these CPEs.

\subsection{Voltammetric behavior of ferrocene and its derivatives}

Cyclic voltammetry of ferrocene and two ferrocene derivatives with negatively and positively charged groups was carried out at the PTFE-PFPE CPE in different electrolyte concentrations. Figure 3 shows the voltammograms of (A) ferrocenecarboxylate $\left(\mathrm{FcCOO}^{-}\right)$, 

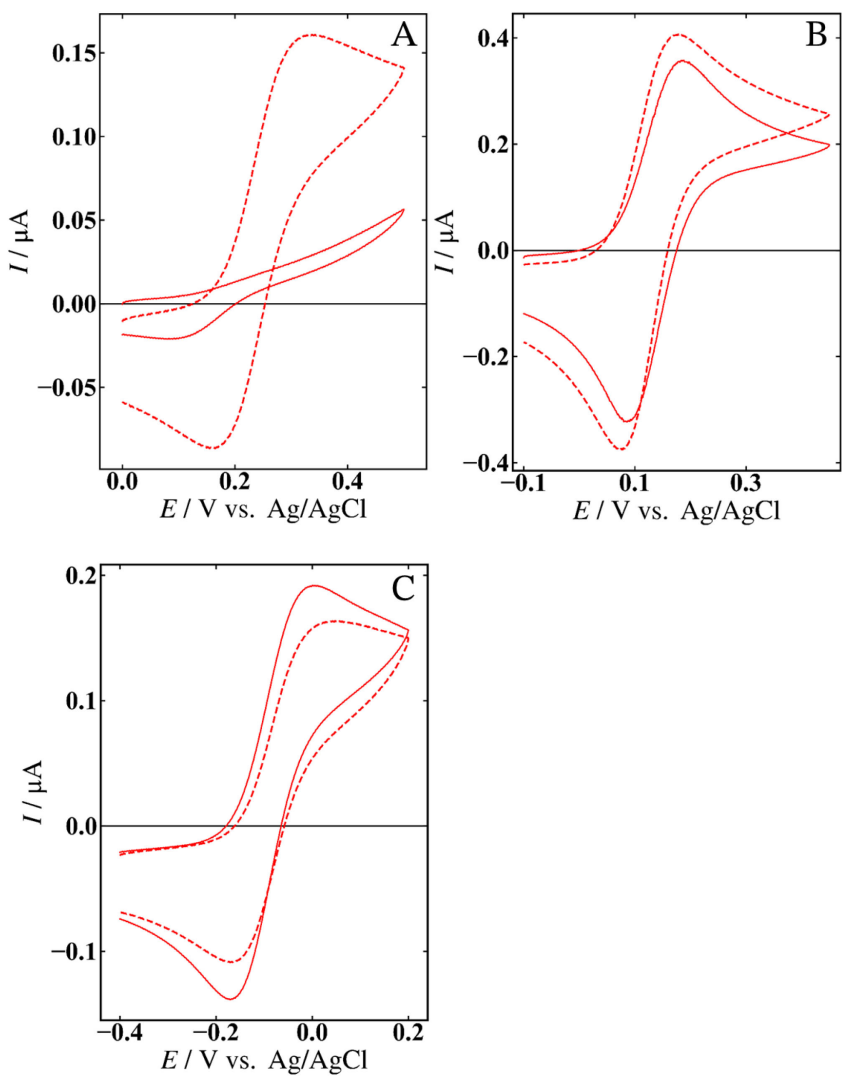

Figure 3. Cyclic voltammograms of (A) $30 \mu \mathrm{M} \mathrm{FcCOO}^{-}$, (B) saturated $\mathrm{Fc}$ and (C) $50 \mu \mathrm{M} \mathrm{FcNH}{ }_{3}{ }^{+}$in acetate buffer (pH 5.1) at PTFE-PFPE CPE. Broken and solid curves indicate the buffer concentrations of $0.1 \mathrm{M}$ and $1 \mathrm{mM}$, respectively. Scan rate: $0.05 \mathrm{~V} \mathrm{~s}^{-1}$.

(B) ferrocene ( $\mathrm{Fc})$, and (C) ferroceneammonium $\left(\mathrm{FcNH}_{3}{ }^{+}\right)$in $0.1 \mathrm{M}$ (broken curves) and $1 \mathrm{mM}$ (solid curves) acetate buffer ( $\mathrm{pH}$ 5.1). At this $\mathrm{pH}$, the carboxy and amino groups of $\mathrm{FcCOO}^{-}$and $\mathrm{FcNH}_{3}{ }^{+}$ were negatively and positively charged, respectively, since the $\mathrm{p} K_{\mathrm{a}}$ of $\mathrm{FcCOOH}$ is $4.2,{ }^{23}$ and that of $\mathrm{FcNH}_{3}{ }^{+}$is $5.9 .{ }^{24}$ A qausi-reversible wave with a pair of anodic and cathodic current peaks of $\mathrm{FcCOO}^{-}$ was obtained in $0.1 \mathrm{M}$ buffer (broken curve in Fig. 3A), whereas a very broadened voltammetric signal with no anodic peak was observed in $1 \mathrm{mM}$ buffer (solid curve in Fig. 3A). No such difference in the shape of voltammograms for different buffer concentrations was seen with $\mathrm{Fc}$ (Fig. 3B). Interestingly, $\mathrm{FcNH}_{3}{ }^{+}$ showed the opposite tendency to $\mathrm{FcCOO}^{-}$, in which the voltammogram obtained in $1 \mathrm{mM}$ buffer had about $50 \mathrm{mV}$ smaller peak potential separation than that in $0.1 \mathrm{M}$ buffer (Fig. 3C). These results suggest that the electrode kinetics is inhibited for negatively charged species and promoted for positively charged species at low electrolyte concentrations, while the electrostatic interaction is shielded and thus the effect is insignificant at high electrolyte concentrations. Also, the clear cathodic peak observed in the reverse scan of $\mathrm{FcCOO}^{-}$in $1 \mathrm{mM}$ buffer (solid curve in Fig. 3A) suggests that the reactant of the reverse scan, $\mathrm{Fc}^{+} \mathrm{COO}^{-}$, is not subject to the electrostatic repulsion, since it has no net charge. These results can be explained by the Frumkin's double-layer effect on electrode kinetics. The fluorine-rich electrode surface with PTFE powder and PFPE oil has localized negative charges due to the high electronegativity of fluorine atom, which may cause the repulsion for anions and the attraction for cations, when the ionic strength is sufficiently low. Similar results have been reported on the effect of fluorination of carbon electrodes on the electrode kinetics. ${ }^{5-7}$ Although the fluorination of the present CPE surface is unlike the

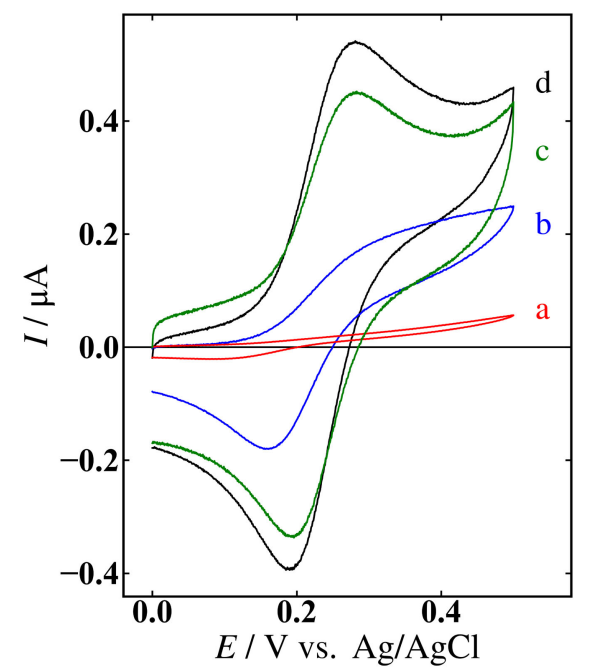

Figure 4. Cyclic voltammograms of $30 \mu \mathrm{M} \mathrm{FcCOO}^{-}$in $1 \mathrm{mM}$ acetate buffer (pH 5.1) at (a) PTFE-PFPE, (b) PTFE-LP, (c) PEPFPE, and (d) PE-LP CPEs. Scan rate: $0.05 \mathrm{~V} \mathrm{~s}^{-1}$.

sputtering technique and more discrete in the scale of $\mu \mathrm{m}$, it may still have an influence on the supply of reactants to the vicinity of the electrode surface. For further discussion, it is important to estimate the point of zero charge (PZC), but it is difficult for the present CPEs. It may be possible to determine the PZC of a dropping carbon fluid electrode $\mathrm{e}^{25-28}$ with fluorinated materials, which will be investigated in our future work. The peak currents of saturated $\mathrm{Fc}$ (Fig. 3B) were considerably larger than those of $\mathrm{FcCOO}^{-}$(Fig. 3A) and $\mathrm{FcNH}_{3}{ }^{+}$(Fig. 3C). Judging from the solubility of $\mathrm{Fc}$ in water, $43 \mu \mathrm{M}$ at $298 \mathrm{~K},{ }^{29}$ there may be some influence of accumulation or adsorption of $\mathrm{Fc}$ on the electrode surface.

The cyclic voltammograms of $\mathrm{FcCOO}^{-}$at the four abovementioned CPEs in $1 \mathrm{mM}$ acetate buffer solution are compared in Fig. 4. The voltammogram with PTFE and LP (curve b) was similar in shape to that with PTFE and PFPE (curve a, the same as the solid curve in Fig. 3A), although the currents were entirely magnified due to the roughness of the electrode surface, as discussed above. Welldefined reversible waves of $\mathrm{FcCOO}^{-}$were obtained with $\mathrm{PE}$ powder (curves $\mathrm{c}$ and $\mathrm{d}$ ). These results indicate that PTFE powder is the predominant factor in the double-layer effect over PFPE oil.

\subsection{Electrochemical measurements in nonaqueous solvent}

In general, CPEs are not suitable for the use in nonaqueous solvents, because the binder liquid can dissolve in the solvents, and the composition of the electrode surface may be varied. ${ }^{30,31}$ However, PFPE is immiscible in most of nonaqueous solvents, including polar aprotic solvents, such as THF. Figure 5 shows the voltammogram of Fc in THF at the PTFE-PFPE CPE. Although relatively large current signal (dotted curve) was observed just after the electrode was immersed in the THF solution, rather reproducible results were obtained in the successive measurements up to $1 \mathrm{~h}$ after the first measurement. The measurements with the other three CPEs were impossible, because they dissolved in THF immediately.

\section{Conclusion}

Voltammetric behavior of the CPEs with fluorinated materials has been studied. Basically, they are like the fluorinated carbon electrodes prepared by sputtering technique, but the present fluorinated CPEs have advantage of being prepared simply by mixing without special instruments for fluorination. The electrostatic interaction between the fluorinated CPE and the charged reactant 


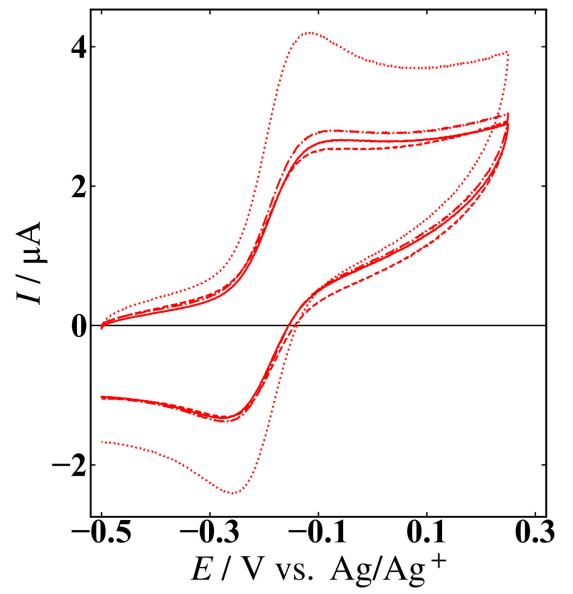

Figure 5. Cyclic voltammograms of $0.16 \mathrm{mM} \mathrm{Fc}$ in $0.1 \mathrm{M}$ $\mathrm{TBAClO}_{4}$ THF solution at PTFE-PFPE CPE. Scan rate: $0.2 \mathrm{~V} \mathrm{~s}^{-1}$. The resting times were $(\cdots \ldots) 0 \mathrm{~min},(---) 20 \mathrm{~min},(-\cdot-) 40 \mathrm{~min}$, and $(-) 60 \mathrm{~min}$ after the electrode was immersed in the THF solution.

affects the electrode kinetics in aqueous solutions at low electrolyte concentrations. It is possible to apply them to nonaqueous solutions in which binder liquids of conventional CPEs such as liquid paraffin and silicone oil may dissolve.

In the present study, we have only examined PTFE and PFPE as fluorinated compounds, but it will be interesting to extend our work to other materials.

\section{Acknowledgments}

The authors are grateful to $3 \mathrm{M}$ Japan and Sumitomo Seika Chemicals for their kind supply of the materials.

\section{References}

1. Fluorine-Carbon and Fluoride-Carbon Materials: Chemistry, Physics, and Applications (Ed. T. Nakajima), Marcel Dekker, New York (1995).

2. T. Nakajima, J. Fluorine Chem., 149, 104 (2013).

3. N. L. Pocard, D. C. Alsmeyer, R. L. McCreery, T. X. Neenan, and M. R. Callstrom, J. Mater. Chem., 2, 771 (1992)

4. H. D. Hutton, W. Huang, D. C. Alsmeyer, J. Kometani, R. L. McCreery, T. X. Neenan, and M. R. Callstrom, Chem. Mater, 5, 1110 (1993).

5. A. Ueda, D. Kato, N. Sekioka, T. Kamata, R. Kurita, H. Uetsuka, Y. Hattori, S. Hirono, S. Umemura, and O. Niwa, Carbon, 47, 1943 (2009).

6. T. Kondo, H. Ito, K. Kusakabe, K. Ohkawa, K. Honda, Y. Einaga, A. Fujishima, and T. Kawai, Diamond Relat. Mater., 17, 48 (2008).

7. C. Yamaguchi, K. Natsui, S. Iizuka, Y. Tateyama, and Y. Einaga, Phys. Chem. Chem. Phys., 21, 13788 (2019).

8. B. Guan, J. Zhi, X. Zhang, T. Murakami, and A. Fujishima, Electrochem. Commun., 9, 2817 (2007)

9. S. Ferro and A. De Battisti, Anal. Chem., 75, 7040 (2003).

10. R. N. Adams, Anal. Chem., 30, 1576 (1958).

11. I. Švancara, K. Kalcher, A. Walcarius, and K. Vytřas, Electroanalysis with Carbon Paste Electrodes, CRC Press, New York, p. 52 (2012).

12. W. Matuszewski and M. Trojanowicz, Analyst, 113, 735 (1988).

13. J. Wang and M. S. Lin, Electroanalysis, 1, 43 (1989).

14. M. Bonakdar, J. L. Vilchez, and H. A. Mottola, J. Electroanal. Chem., 266, 47 (1989).

15. J. Lindquist, J. Electroanal. Chem., 52, 37 (1974).

16. N. D. Danielson, J. Wangsa, and M. A. Targove, Anal. Chem., 61, 2585 (1989).

17. J. Wangsa and N. D. Danielson, J. Chromatogr., A, 514, 171 (1990).

18. J. Wangsa and N. D. Danielson, Electroanlaysis, 3, 625 (1991).

19. J. E. Anderson, D. E. Tallman, D. J. Chesney, and J. L. Anderson, Anal. Chem., 50, 1051 (1978)

20. L. N. Klatt, D. R. Connell, R. E. Adams, I. L. Honigberg, and J. C. Price, Anal. Chem., 47, 2470 (1975).

21. C. Olson and R. N. Adams, Anal. Chim. Acta, 29, 358 (1963).

22. CRC Handbook of Chemistry and Physics (95th ed.), CRC Press, New York, p. 13-17 (2014).

23. T. Matsue, D. H. Evans, T. Osa, and N. Kobayashi, J. Am. Chem. Soc., 107, 3411 (1985).

24. J. Věžník, M. Konhefr, L. Trnková, P. Skládal, and K. Lacina, Electrochim. Acta, 318, 534 (2019).

25. H. Tatsumi and M. Shiba, Electrochem. Commun., 20, 160 (2012).

26. H. Tatsumi and S. Tanaka, Electrochim. Acta, 135, 255 (2014).

27. H. Tatsumi, N. Seike, and K. Kubo, J. Electroanal. Chem., 779, 236 (2016).

28. T. Okada, K. Kubo, and H. Tatsumi, J. Electroanal. Chem., 854, 113526 (2019).

29. J. Wu, K. Toda, A. Tanaka, and I. Sanemasa, Bull. Chem. Soc. Jpn., 71, 1615 (1998).

30. R. N. Adams, Rev. Polarogr., 11, 71 (1963).

31. L. S. Marcoux, K. B. Prater, B. G. Prater, and R. N. Adams, Anal. Chem., 37, 1446 (1965). 